\title{
Differences of HBME-1 Expression in Thyroid Follicular Adenoma and Follicular Thyroid Carcinoma by Ultrasound-Guided Fine-Needle Aspiration Biopsy
}

\author{
Junsong Wang ${ }^{1}$, Li Xu2 ${ }^{2}$ Xiaosen $\mathrm{Xu}^{3}$ and Xin Wang ${ }^{4}$
}

\begin{abstract}
Objective: To investigate the differences in human bone marrow endothelial cell marker 1 (HBME-1) expression in thyroid follicular adenoma (FA) and follicular thyroid carcinoma (FTC) by ultrasound-guided fine-needle aspiration biopsy.

Study Design: An observational study.

Place and Duration of Study: The Department of Electrical Diagnosis, The Second Hospital of Jilin University, Jilin Province, China, from January 2017 to April 2018.

Methodology: Forty-five patients with FTC were selected as FTC group, and 45 patients with FA were selected as FA group. Ultrasound-guided fine-needle aspiration biopsy specimens from two groups were analyzed for HBME-1 expression. Sensitivity, specificity, and accuracy of HBME-1 expression by ultrasound-guided fine-needle aspiration biopsy specimens in FTC group were analyzed using HBME-1 expression in the pathological tissues as criteria.

Results: The positive expression rate of HBME-1 in ultrasound-guided fine-needle aspiration biopsy specimens was higher in the FTC group than that in the FA group $(p=0.003)$. The sensitivity, specificity, and accuracy of HBME-1 expression by ultrasound-guided fine-needle aspiration biopsy specimens in the FTC group were $77.78 \%$ (28/36), $88.89 \%$ (8/9) and $80.00 \%(36 / 45)$, respectively.

Conclusion: HBME-1 detection with ultrasound-guided fine-needle aspiration biopsy may be useful clinically to pick out cases with follicular thyroid carcinoma, and that thyroid follicular adenoma cases need close follow-up.
\end{abstract}

Key Words: Ultrasound-guided fine-needle aspiration biopsy, HBME-1, Follicular thyroid carcinoma, Thyroid follicular adenoma.

\section{INTRODUCTION}

Follicular thyroid carcinoma (FTC) is the most common malignancy of thyroid cancers except papillary carcinoma. 1 The traditional method for diagnosing FTC is mainly based on the penetration of the tumor capsule and vascular invasion, but the preoperative biopsies cannot observe the whole tumor capsule and blood vessel conditions even if those are taken from multiple points. .,3 $^{2}$ Moreover, the well-differentiated FTC and thyroid follicular adenoma (FA) are histologically similar and difficult to distinguish. ${ }^{4}$ Therefore, in order to improve the preoperative diagnosis of FTC, more scholars focus on finding suitable molecular markers to

1 Department of Electrical Diagnosis / Laboratory Medicine ${ }^{2}$, The Second Hospital of Jilin University, Jilin Province, 130041, China

3 Department of Electrical Diagnosis, Changchun Traditional Chinese Medicine Hospital, Jilin Province, 130000, China

${ }_{4}$ Department of Laboratory Medicine, The First Clinical Hospital of Bethune Medical, Jilin University, Jilin Province, 130021, China

Correspondence: Xin Wang, Department of Laboratory Medicine, The First Clinical Hospital of Bethune Medical, Jilin University, Jilin Province, 130021, China

E-mail:fopon7@163.com

Received: May 24, 2018; Accepted: October 29, 2018 assist in the differential diagnosis of FTC. ${ }^{5}$ Human bone marrow endothelial cell marker 1 (HBME-1) is an antigen component of microvilli on the surface of the mesothelium. It is often used for diagnosis of mesothelial origin tumors. ${ }^{6}$ In recent years, HBME- 1 has gradually been used for the diagnosis of thyroid cancer.7,8 Moyano et al. reported that HBME-1 was highly expressed in papillary thyroid carcinoma. ${ }^{9}$ Current research is mostly based on the detection of HBME-1 in thyroid cancer on the basis of tissue expression, which is less convenient than ultrasound-guided fine-needle aspiration biopsy. ${ }^{10}$ However, there are few studies on whether the expression of HBME-1 in ultrasound-guided fine-needle aspiration biopsy specimens from patients with FTC is significantly different from those with FA. As a result, it lacks basis to determine whether to detect HBME-1 expression in patients with ultrasound-guided fineneedle aspiration biopsy instead of biopsy.

The objective of this study was to investigate the differences of HBME-1 expression in FA and FTC by ultrasound-guided fine-needle aspiration biopsy.

\section{METHODOLOGY}

This observational study was conducted at the Department of Electrical Diagnosis, The Second Hospital of Jilin University, Jilin Province, China, from January 2017 to April 2018. The study was conducted 
Table I: Comparison of HBME-1 expression in ultrasound-guided fine-needle aspiration biopsy specimens between two groups.

\begin{tabular}{|c|c|c|c|c|c|c|c|c|c|c|c|c|}
\hline \multirow[t]{2}{*}{ Groups } & \multirow[t]{2}{*}{$\mathrm{n}$} & \multicolumn{2}{|l|}{-} & \multicolumn{2}{|l|}{+} & \multicolumn{2}{|l|}{++} & +++ & \multicolumn{3}{|c|}{$\begin{array}{l}\text { Positive } \\
\text { expression } \\
\text { of HBME-1 }\end{array}$} & \multirow[t]{2}{*}{$p$-value } \\
\hline & & $\mathrm{n}$ & $\%$ & $\mathrm{n}$ & $\%$ & $n$ & $\%$ & $\mathrm{n}$ & $\%$ & $\mathrm{n}$ & $\%$ & \\
\hline FA group & 45 & 30 & 66.67 & 9 & 20.00 & 4 & 8.89 & 2 & 4.44 & 15 & 33.33 & 0.003 \\
\hline FTC group & 45 & 16 & 35.56 & 14 & 31.11 & 10 & 22.22 & 5 & 11.11 & 29 & 64.44 & \\
\hline
\end{tabular}

after approval from the Hospital Ethical and Research Committee. Forty-five patients with FTC (45 nodules) were selected as FTC group, and 45 patients with FA (45 nodules) were selected as FA group. Inclusion criteria were ultrasound-guided fine-needle aspiration biopsy performed before surgery and specimens stored intact for detecting HBME-1 expression; nodules diagnosed as FTC or FA with detection of HBME-1 expression performed. Patients were preliminarily diagnosed and underwent surgical treatment in this hospital without treatment like any other endocrine therapy, radiotherapy or chemotherapy. Exclusion criteria were patients with other serious diseases, and incomplete record of clinical and/or accessory examinations. Both FTC group and FA group underwent ultrasound examination to determine the puncture nodules. All patients were placed in supine head-down position, lying toward the non-puncture side to expose the puncture site. After sterilization and local anesthesia, under the guidance of ultrasound, the needle was connected to the $10 \mathrm{~mL}$ syringe and pierced the skin at an angle of about $45^{\circ}$. The pintle was pushed back and forth, and negative pressure was inserted in different directions for five times. The aspirate was smeared and fixed. Then it was sent to the pathology department for HBME-1 detecting and reading. The ultrasound suspicious signs, such as microcalcification, was taken during puncture.

Immunohistochemical streptavidin-perosidase (SP) method was used to detect the expression of HBME-1 in pathological nodule tissue of FTC patients. Tissue specimens were formalin-fixed before following the steps of paraffin embedding, sectioning, dewaxing and hydration. Two highly-qualified pathologists performed microscopic examination to determine the staining results. Judging standard was positive staining of HBME-1, was found in the tumor cell membrane and the follicular gland margin or stained in the cytoplasm.11 Staining intensity scoring was marked 0 point for no coloring, 1 point for light yellow, 2 points for brown, and 3 points for tan. Positive distribution range scoring was considered $<5 \%$ for negative, $5 \%$ to $25 \%$ for 1 point, $26 \%$ to $50 \%$ for 2 points, $51 \%$ to $75 \%$ for 3 points, and $>75 \%$ for 4 points. Both scores were multiplied; 0 point given for negative (-), 1 to 2 points for weak positive $(+)$, 3 to 6 points for moderate positive $(++)$, and $>6$ points for strong positive $(+++) .12$

Differences of HBME-1 expression in ultrasound-guided fine-needle aspiration biopsy specimens were analyzed

between patients in the FTC group and the FA group. The sensitivity, specificity, and accuracy of HBME-1 expression by ultrasound-guided fine-needle aspiration biopsy specimens in the FTC group were analyzed using HBME-1 expression in the pathological tissues of patients in the FTC group as criteria.

Data was entered and analyzed by using SPSS version 25.0. Count data was indicated in $n(\%)$, and $X^{2}$ test was used to compare the outcomes between two groups. $P$-value of less than 0.05 was considered significant.

\section{RESULTS}

In FTC group, there were 17 males $(37.78 \%)$ and 28 females (62.22\%); aged $26-71$ years, with an average age of $54.76 \pm 3.83$ years. In FA group, there were 16 males $(35.56 \%)$ and 29 females $(64.44 \%)$; aged $28-72$ years, with an average age of $55.15 \pm 4.04$ years.

The positive expression rate of HBME-1 in ultrasoundguided fine-needle aspiration biopsy specimens was higher in the FTC group than that in the FA group $(p=0.003$, Table I). Immunohistochemical detection of pathological nodule tissue of HBME-1 in the FTC group was shown in Figures 1 and 2.

Table II: Expression of HBME-1 in pathological nodular tissue and ultrasound-guided fine-needle aspiration biopsy specimens of FTC patients.

\begin{tabular}{l|cc}
\hline \multirow{2}{*}{ Item } & \multicolumn{2}{|c}{ Expression of HBME-1 in pathological nodular tissue } \\
\cline { 2 - 3 } & Positive (cases) & Negative (cases) \\
\hline $\begin{array}{l}\text { Expression of HBME-1 } \\
\text { in ultrasound-guided } \\
\text { fine-needle aspiration }\end{array}$ & & \\
biopsy specimens & & \\
$\quad$ Positive (cases) & 28 & \\
$\quad$ Negative (cases) & 8 & 8 \\
\hline Sensitivity (\%) & $77.78(28 / 36)$ \\
Specificity (\%) & $88.89(8 / 9)$ & \\
Accuracy (\%) & $80.00(36 / 45)$ & \\
\hline
\end{tabular}

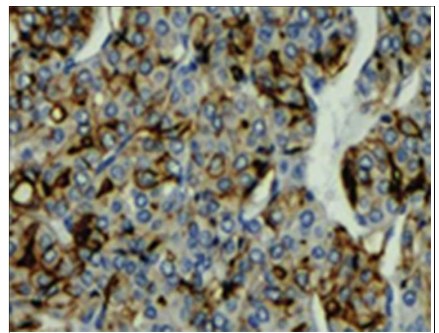

Figure 1: Positive expression of HBME-1 in pathological nodules of patients with FTC.

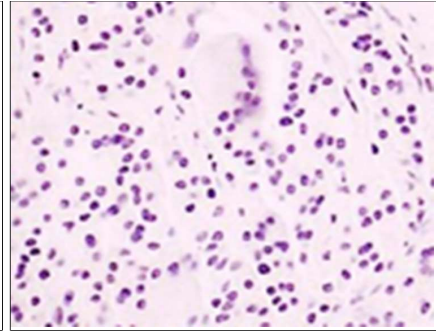

Figure 2: Negative expression of HBME-1 in pathological nodules of patients with FTC. 
HBME-1 was positively expressed in 36 cases (80.00\%) of pathological nodules in the FTC group and negatively expressed in 9 cases $(20.00 \%)$. The sensitivity, specificity, and accuracy of HBME-1 expression by ultrasoundguided fine-needle aspiration biopsy specimens in the FTC group were $77.78 \%(28 / 36), 88.89 \%(8 / 9)$ and $80.00 \%$ (36/45), respectively, using HBME-1 expression in the pathological tissues as criteria, shown in Table II.

\section{DISCUSSION}

Thyroid cancer is the most common cervical malignancy. Early detection and early treatment have important clinical implications for the treatment of thyroid cancer. In thyroid cancer, the incidence of thyroid papillary carcinoma is the highest, but its prognosis is good. The incidence of FTC is second only to thyroid papillary carcinoma, but the prognosis is poor. Moreover, FTC lacks a special tissue structure and cell morphology, and differently differentiated follicles can be seen under the microscope, while FA is a benign tumor with complete envelope and follicular differentiation under the microscope. ${ }^{13,14}$ Therefore, some FTCs with well differentiated follicles are difficult to be distinguished from adenomas. 15 The differential diagnosis of FTC and FA before surgery is a clinical challenge for even an experienced pathologist, and requires special effort. 16

Diagnosis of FTC, based on tumor capsule penetration, vascular invasion or distant metastasis has certain limitations. It can also lead to misdiagnosis due to improper sampling and slicing. This has led to the fact that some patients with benign tumors underwent surgery required only for malignant tumors, or a partially well-differentiated FTC was misdiagnosed as FA, which in turn led to failure of timely and appropriate treatment for FTC patients. $17 \mathrm{Omi}$ et al. pointed out that some patients are initially diagnosed as FA but develop metastases, indicating the original lesion was FTC. ${ }^{18}$ Therefore, the study of the pathogenesis of thyroid follicular epithelium-derived tumors has received increasing attention. It is hoped that suitable auxiliary means can be found to assist in the differential diagnosis of FTC.

HBME-1 is an acidic amino-acid polysaccharide protein, composed mainly of hyaluronic acid and other components. ${ }^{19}$ Clinical studies have shown that HBME-1 is highly expressed in papillary thyroid carcinoma and has a good diagnostic value for thyroid cancer. 20 Coli et al. revealed that HBME-1 could be used as a detection index for ultrasound-guided fine-needle aspiration biopsy specimens of thyroid nodules and can improve the sensitivity and specificity of thyroid tumor diagnosis. 21 The use of tissue for the detection of HBME-1 expression in the past had demonstrated a significant difference between FTC and FA. ${ }^{22}$ However, due to the fact that biopsy may cause bleeding and other risks, ultrasound-guided fine-needle aspiration biopsy instead of biopsy was used for preoperative examination. In this study, it was found that HBME-1 was expressed in both FTC and FA in ultrasound-guided fine-needle aspiration biopsy specimens, and there was a significant difference between the two groups.

The results of this study showed that the sensitivity, specificity, and accuracy of HBME-1 in ultrasoundguided fine-needle aspiration biopsy specimens were $77.78 \%, 88.89 \%$ and $80.00 \%$, respectively. It is suggested that ultrasound-guided fine-needle aspiration biopsy specimens of patients with FTC can well reflect the expression of HBME-1. Therefore, the authors believe that in the use of ultrasound-guided fine-needle aspiration biopsy specimens of follicular thyroid tumors for the detection of HBME-1 expression, the greater positive expression cases should be more considered for FTC.

The number of relevant cases included in this study is not large, so it may be difficult to comprehensively compare the value of HBME-1 in diagnosing FTC. Further studies are needed to be done in the future.

\section{CONCLUSION}

HBME-1 detection with ultrasound-guided fine-needle aspiration biopsy may be useful clinically to pick out cases with follicular thyroid carcinoma, and that thyroid follicular adenoma cases need close follow-up.

\section{REFERENCES}

1. Nicolson NG, Murtha TD, Dong W, Paulsson JO, Choi J, Barbieri AL, et al. Comprehensive genetic analysis of follicular thyroid carcinoma predicts prognosis independent of histology. $J$ Clin Endocrinol Metab 2018; 103:2640-50.

2. Mishra A, Kumar C, Chand G, Agarwal G, Agarwal A, Verma AK, et al. Long-term outcome of follicular thyroid carcinoma in patients undergoing surgical intervention for skeletal metastases. World J Surg 2016; 40:562-9.

3. Stenson G, Nilsson IL, Mu N, Larsson C, Lundgren Cl, Juhlin CC, et al. Minimally invasive follicular thyroid carcinomas: prognostic factors. Endocrine 2016; 53:505-11.

4. Sponziello M, Lavarone E, Pegolo E, Di LC, Puppin C, Russo MA, et al. Molecular differences between human thyroid follicular adenoma and carcinoma revealed by analysis of a murine model of thyroid cancer. Endocrinology 2013; 154: 3043-53.

5. Brown C, Mangano W, Thompson S, Richmond B. Factors predicting thyroid malignancy in fine needle aspiration biopsy specimens classified as atypia of uncertain significance/ follicular lesion of uncertain significance. Am Surg 2018; 84: 1207-13.

6. Abu-Sinna E, Hasan MY, El-Deftar MM, Amer SA, Abdelsalam LO, Nakhla JA. Galectin-3 and HBME-1 expression on agarose cell blocks from fine-needle aspirates of follicular cell-derived thyroid tumors. J Cytol 2018; 35:27-32.

7. Murtezaoglu AR, Gucer H. Diagnostic value of TROP-2 expression in papillary thyroid carcinoma and comparison with HBME-1, galectin-3 and cytokeratin 19. Pol J Pathol 2017; 68: 1-10. 
8. Arcolia V, Journe $F$, Renaud $F$, Leteurtre $E$, Gabius $H J$, Remmelink M, et al. Combination of galectin-3, CK19 and HBME-1 immunostaining improves the diagnosis of thyroid cancer. Oncol Lett 2017; 14:4183-9.

9. Moyano L, Franco C, Carreño L, Robinson P, Sánchez G. HBME-1 and cyclin D1 as diagnostic markers for follicular thyroid carcinoma. Rev Med Chil 2004; 132:279-84.

10. Cheah WK. Thyroid cancer: diagnosis and management. Singapore Med J 2007; 48:107-12.

11. Lenggenhager $D$, Maggio EM, Moch H, Rössle M. HBME-1 expression in hyalinizing trabecular tumours of the thyroid gland. Histopathology 2013; 62:1092-7.

12. Tollenaar RA, van Krieken JH, van Slooten HJ, Bruinvels DJ, Nelemans KM, van den Broek LJ, et al. Immunohistochemical detection of p53 and $\mathrm{Bcl}-2$ in colorectal carcinoma: No evidence for prognostic significance. Br J Cancer 1998; 77:1842-7.

13. Liu Z, Zhou G, Nakamura M, Koike E, Li Y, Ozaki T, et al. Encapsulated follicular thyroid tumor with equivocal nuclear changes, so-called well-differentiated tumor of uncertain malignant potential: A morphological, immunohistochemical, and molecular appraisal. Cancer Sci 2011; 102:288-94.

14. Jeong SH, Hong HS, Lee EH. Can nodular hyperplasia of the thyroid gland be differentiated from follicular adenoma and follicular carcinoma by ultrasonography? Ultrasound Q 2016; 32:349-55

15. Cossu A, Lissia A, Dedola MF, Deiana A, Faedda R, Palmieri G, et al. Classic follicular dendritic reticulum cell tumor of the lymph node developing in a patient with a previous inflammatory pseudotumor-like proliferation. Hum Pathol 2005; 3:207-11.

16. Pfeifer A, Wojtas B, Oczko-Wojciechowska M, Kukulska A, Czarniecka A, Eszlinger $\mathrm{M}$, et al. Molecular differential diagnosis of follicular thyroid carcinoma and adenoma based on gene expression profiling by using formalin-fixed paraffinembedded tissues. BMC Med Genomics 2013; 6:38.

17. Rezk S, Khan A. Role of immunohistochemistry in the diagnosis and progression of follicular epithelium-derived thyroid carcinoma. Appl Immunohistochem Mol Morphol 2005; 13:256-64.

18. Omi Y, Shibata N, Okamoto T, Obara T, Kobayashi M. The role of CD147 in the invasiveness of follicular thyroid carcinoma cells. Thyroid 2012; 22:383-94.

19. De Matos PS, Ferreira AP, De OFF, Assumpção LV, Metze K, Ward LS. Usefulness of HBME-1, cytokeratin 19 and galectin-3 immunostaining in the diagnosis of thyroid malignancy. Histopathology 2005; 47:391-401.

20. Casey MB, Lohse CM, Lloyd RV. Distinction between papillary thyroid hyperplasia and papillary thyroid carcinoma by immunohistochemical staining for cytokeratin 19, galectin-3, and HBME-1. Endocr Pathol 2003; 14:55-60.

21. Coli A, Bigotti G, Parente P, Federico F, Castri F, Massi G. Atypical thyroid nodules express both HBME-1 and galectin-3, two phenotypic markers of papillary thyroid carcinoma. J Exp Clin Cancer Res 2007; 26:221-7.

22. Zhu X, Sun T, Lu H, Zhou X, Lu Y, Cai X, et al. Diagnostic significance of CK19, RET, galectin-3 and HBME-1 expression for papillary thyroid carcinoma. J Clin Pathol 2010; 63:786-9. 